\title{
DERIVATION OF ASYMPTOTIC FREQUENCY EQUATIONS IN TERMS OF RAY AND NORMAL MODE THEORY AND SOME RELATED PROBLEMS- RADIAL AND SPHEROIDAL OSCILLATIONS OF AN ELASTIC SPHERE
}

\author{
Toshikazu OdAKA \\ Earthquake Research Institute, University of Tokyo, Tokyo, Japan
}

(Received August 8, 1977; Revised January 26, 1978)

\begin{abstract}
Ray theory is applied successfully to derive asymptotic frequency equations of the radial oscillations of a homogeneous elastic sphere and a sphere with one concentric surface of discontinuity in it, and of the spheroidal oscillation of a homogeneous sphere. The method is similar to the one used for getting frequency equations for plane stratified media, and is based on the idea that for steady vibration of a sphere to be possible some interference condition has to be satisfied by body waves traveling in it. It is shown that these equations are identical to those obtained by the normal mode theory.

It is found that the solotone effect has an immediate connection with multiple reflections of body waves at the discontinuity in the medium.

Decoupling of $\mathrm{P}_{-}$and $\mathrm{S}$-waves at high frequencies is well illustrated for the spheroidal modes in terms of the distribution of eigenfrequencies and the radial dependence of eigenfunctions.
\end{abstract}

\section{Introduction}

It is known that for a plane stratified medium a frequency equation which governs the periods of normal mode oscillations of the medium can be interpreted as an interference phenomenon of relevant body-wave rays (PRESS and Ewing, 1948; OfFicer, 1951; SAtô, 1951; Tolstoy and Usdin, 1953; Tolstoy, 1954). For a spherical body, some investigations have revealed mode-ray correspondence relations. Ben-MENAHEM (1964) interpreted an eigenvalue solution for a sphere in terms of ray geometry by expanding it in an asymptotic form. BRUNE (1964) introduced an interference condition of surface waves and body waves in a sphere and tried to get dispersion curves of toroidal modes from the analysis of body waves. ODAKA and UsAMI (1972) 
found some correspondence relations between torsional modes and SH-rays, and demonstrated numerically that a body wave phase having any specified ray parameter is contributed to by only a limited number of modes at each branch of a given radial mode number. Recently, SATO and LAPWOOD (1977) gave thorough discussions on asymptotic properties of radial overtones in torsional eigenfrequencies of layered spheres and interpreted the frequency equations in terms of ray geometry. However, there is no established method for spherical problems of deriving frequency equations directly from raytheoretical considerations.

In this paper we make such an attempt for the purely radial oscillation of an elastic sphere with one concentric surface of discontinuity in it and for the spheroidal oscillation of a homogeneous sphere, and compare them with asymptotic frequency equations obtained analytically from normal mode solutions.

\section{Radial Oscillation of an Elastic Sphere with One Surface of Discontinuity}

The radial oscillation can be classified as a special case of the spheroidal oscillation. It is better, however, to treat the two oscillations separately, because in the former case the whole movement in a sphere is confined to the radial direction, and the only body waves concerned with this oscillation are $\mathrm{P}$-waves traveling through the center of the sphere; thus the problem becomes very simple.

Here, we consider an elastic sphere consisting of a homogeneous inner sphere of radius $b$ and a homogeneous spherical shell of inner and outer radii $b$ and $a(>b)$ in welded contact (see Fig. 1). A homogeneous sphere is included in the above model as a special case.

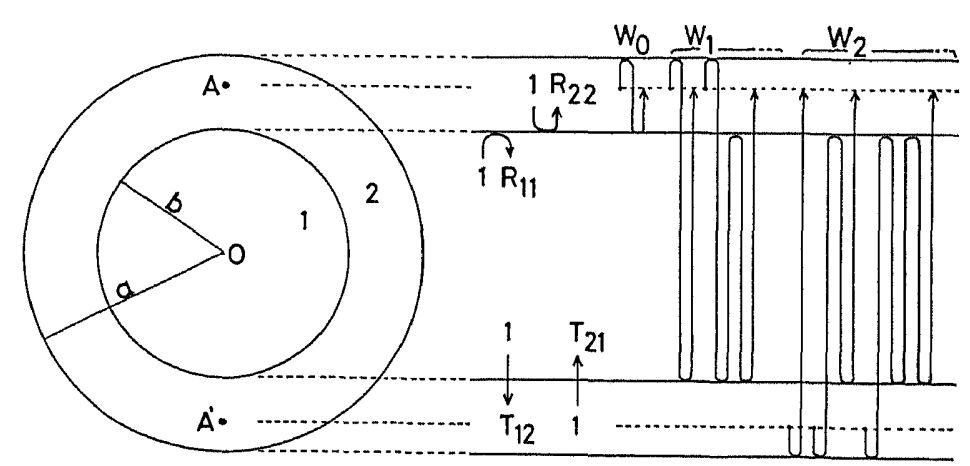

Fig. 1. Three types of outgoing waves at a point $A$. The disturbance of a wave going outwards at $A$ results from a summation of contributions from all these waves. 
Tolstoy and UsDin (1953) constructed characteristic equations determining dispersive properties of plane layered media under the condition that media are in steady states. Similarly we suppose that the sphere vibrates steadily. Suppose that A is a point in the outer medium; this point is subject to vibration $\exp (i \omega t)$ under the steady state condition. In ray theory, this situation can be expressed as follows: the disturbance of unit amplitude at $\mathrm{A}$ is constituted from disturbances emanating with unit amplitudes from $\mathbf{A}$ (or other points equivalent to $\mathrm{A}$ ), multiply reflected in the sphere and arriving again at $\mathrm{A}$. This interference condition ensures that the point $\mathrm{A}$ is subject to persistent steady vibration. Here, we are concerned about waves going outwards at A. It will be found that there exist three types of waves which contribute independently to the disturbance at A. The first one is a wave reflected once at the surface and once at the interface in the sphere (denoted as $W_{0}$ in Fig. 1). The second type consists of waves reflected once at the surface and once, three times or multiply reflected at the interface $\left(W_{1}\right)$. The third group consists of waves starting from a point $\mathrm{A}^{\prime}$ (spherically symmetric with $\mathrm{A}$ ), reflected once at the surface and not reflected or twice or multiply reflected at the interface, which is denoted as $W_{2}$. We have to take account of the point $\mathrm{A}^{\prime}$ as well as $\mathrm{A}$. That becomes clear for rays which are slightly deflected from normal incidence (see Fig. 2). All the other rays outwardly cross points $\mathrm{A}$ and/or $\mathrm{A}^{\prime}$ once at least, and thus any one of those ray-paths connects with one of the above three groups of rays. In consequence the contribution from such a ray is inevitably included in the contributions from $W_{0}, W_{1}$ and $W_{2}$. In Fig. 2, for example, the contribution from the disturbance at $B$ to the disturbance at $A$ is equivalent to contributions from the three groups of rays as denoted by the dashed lines.

The interference condition gives us the following equation in which the amplitude of disturbance on the surface where $A$ and $A^{\prime}$ are situated is normalized to be unity.

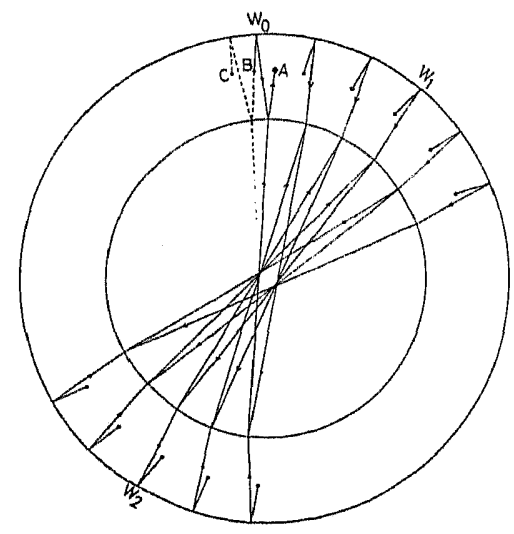

Fig. 2. Three types of rays (solid lines) emanating outwards from points having the same radius as $\mathrm{A}$ and traveling to $\mathrm{A}$. The only difference between two groups of rays, $W_{1}$ and $W_{2}$, is whether the number of times of reflection at the interface is odd or even. 


$$
\begin{aligned}
1= & \left.R_{22} \mathrm{e}^{-i 2 h_{2} d}+R_{11} T_{21} T_{12} \mathrm{e}^{-i\left(2 h_{2} d+4 h_{1} b\right)}+R_{11}^{3} T_{21} T_{12} \mathrm{e}^{-i\left(2 h_{2} d+8 h_{1} b\right.}\right)+\cdots \\
& +T_{21} T_{12} \mathrm{e}^{-i\left(2 h_{2} d+2 h_{1} b\right)}+R_{11}^{2} T_{21} T_{12} \mathrm{e}^{-i\left(2 h_{2} d+8 h_{1} b\right)}+\cdots
\end{aligned}
$$

where

$$
h_{j}=\omega / \alpha_{j} \quad(j=1,2), \quad d=a-b .
$$

$\alpha_{1}$ and $\alpha_{2}$ are the $\mathrm{P}$-wave velocities in the inner sphere and in the outer shell respectively. $R_{i j}$ and $T_{i j}$ are the reflection and transmission coefficients as defined in Fig. 1. At high frequency limits, spherical waves can be regarded approximately as plane waves. Under such a condition, we have for these coefficients

$$
\begin{aligned}
& R_{11}=\left(\rho_{1} \alpha_{1}-\rho_{2} \alpha_{2}\right) / \Delta, \quad T_{12}=2 \rho_{1} \alpha_{1} / \Delta, \quad T_{21}=2 \rho_{2} \alpha_{2} / \Delta, \\
& R_{22}=-R_{11}, \quad R_{11}^{2}+T_{12} T_{21}=1, \quad \Delta=\rho_{1} \alpha_{1}+\rho_{2} \alpha_{2},
\end{aligned}
$$

where $\rho_{1}$ and $\rho_{2}$ are the densities in the respective media. Employing the relations (2.3), Eq. (2.1) can be reduced to

$$
1-\mathrm{e}^{-i\left(2 h_{2} d+2 h_{1} b\right)}-R_{11} \mathrm{e}^{-i 2 h_{1} b}+R_{11} \mathrm{e}^{-i 2 h_{2} d}=0 .
$$

Further reduction yields

$$
\left(\rho_{1} \alpha_{1}+\rho_{2} \alpha_{2}\right) \sin \left(h_{1} b+h_{2} d\right)+\left(\rho_{1} \alpha_{1}-\rho_{2} \alpha_{2}\right) \sin \left(h_{1} b-h_{2} d\right)=0 .
$$

It can be proved that this equation is identical to the asymptotic frequency equation obtained by the normal mode theory. The exact frequency equation is given by

where

$$
\begin{aligned}
\mu_{1}\left\{g\left(j_{0}, h_{2} a\right) \dot{n}_{0}\left(h_{2} b\right)-g\left(n_{0}, h_{2} a\right) \dot{j}_{0}\left(h_{2} b\right)\right\} g\left(j_{0}, h_{1} b\right) \\
-\mu_{2}\left\{g\left(j_{0}, h_{2} a\right) g\left(n_{0}, h_{2} b\right)-g\left(n_{0}, h_{2} a\right) g\left(j_{0}, h_{2} b\right)\right\} \dot{j}_{0}\left(h_{1} b\right)=0
\end{aligned}
$$

$$
\begin{aligned}
\dot{z}_{0}(h r) & =d\left\{z_{0}(h r)\right\} / d r, \\
g\left(z_{0}, h r\right) & =-(4 / r) \dot{z}_{0}(h r)-k^{2} z_{0}(h r),
\end{aligned}
$$

and $\mu$ is the rigidity, $k$ the wave number for S-wave, and $j_{0}$ and $n_{0}$ the spherical Bessel functions of the first and the second kind. The detailed formulation for the excitation of radial oscillations of an $n$-layered spherical model and its application are made by ODAKA (1978). It is easy to show that (2.6) can be transformed to (2.5) at high frequency limits.

Equation (2.5) is quite similar in its form to the frequency equation of a vibrating string consisting of two uniform parts of different densities, which was obtained by LAPWOOD (1975) for discussing the effect of a discontinuity in the medium on the distribution of eigenfrequencies. Equation (2.5) provides discrete eigenfrequencies of unequal spacing, giving rise to the so called solotone effect (MCNABB et al., 1976). The above process of deriving (2.5) makes it clear that the solotone effect has an immediate connection with the 


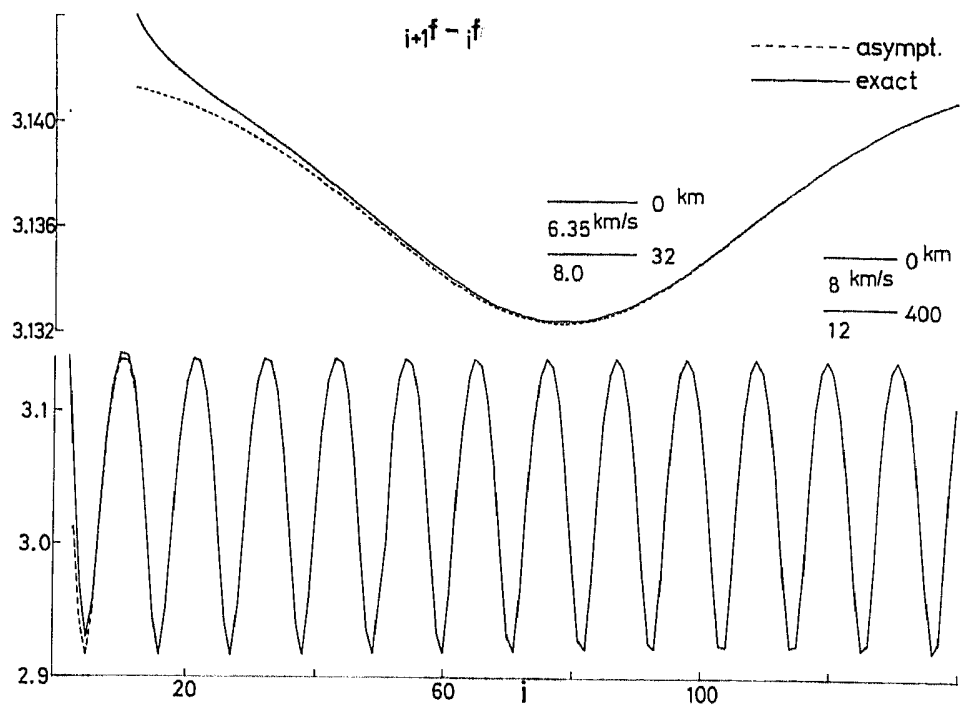

Fig. 3. Difference of two successive eigenfrequencies for the radial oscillation of two earth models as plotted against the radial mode number. Solid and dashed curves are calculated from the exact and asymptotic frequency equations respectively.

phenomenon of multiple reflections of waves at the discontinuity in the medium. WANG et al. (1977) have also reached the same conclusion from somewhat different considerations.

Comparison of solutions of two Eqs. (2.5) and (2.6) is made for two earth models in Fig. 3, where the difference of two successive eigenfrequencies, ${ }_{i+1} f-{ }_{i} f$, is plotted against the radial mode number $i$. Frequencies are given in units of $\alpha_{1} / 2 \pi a$. The solid and broken curves correspond to exact and asymptotic values respectively. The two models are illustrated in the figure, and it is assumed that Poisson's ratio is equal to 0.25 and $\rho_{1}=\rho_{2}$. Oscillatory properties are conspicuous and are strongly dependent on the models. WANG et al. (1977) have investigated in detail the effect of the depth and the scale of discontinuities in the sphere on the asymptotic properties of torsional eigenfrequencies.

For reference, numerical values of exact and asymptotic eigenfrequencies are given for several radial mode numbers in Table 1 . Model 1 corresponds to the top curves of Fig. 3 and Model 2 to the bottom curves respectively. Agreement between two frequencies is extremely good even for relatively low radial modes.

It will be instructive to get a frequency equation for the radial oscillation of a homogeneous sphere. In this case, except for the first ray of the group $W_{2}$ in Fig. 1, all the other rays vanish, and we have 
Table 1. Comparison of exact and asymptotic eigenfrequencies (unit: $\alpha_{1} / 2 \pi a$ ) for the radial oscillation of two earth models.

\begin{tabular}{rcccccc}
\hline & \multicolumn{2}{c}{ Model 1 } & & \multicolumn{2}{c}{ Model 2 } \\
\cline { 6 - 7 } \cline { 5 - 6 }$i$ & $f_{\text {exact }}$ & $f_{\text {asympt }}$ & & $f_{\text {exact }}$ & $f_{\text {asy mpt }}$ \\
\hline 10 & 31.372498 & 31.415157 & & 30.326155 & 30.371493 \\
20 & 62.804394 & 62.825683 & & 60.737701 & 60.760036 \\
50 & 156.97607 & 156.98456 & & 152.19771 & 152.20590 \\
100 & 313.65397 & 313.65821 & & 304.72007 & 304.72457 \\
\hline
\end{tabular}

$$
1=\mathrm{e}^{-i 2 h a}
$$

where

$$
h=\omega / \alpha,
$$

and $\alpha$ is the $P$-wave velocity in the sphere. Equation (2.8) is identical to

$$
\sin h a=0 \text {. }
$$

An exact frequency equation is, on the other hand, given by

$$
g\left(j_{0}, h a\right)=0 \text {. }
$$

At high frequency limits, this equation can be reduced to (2.10). Unlike Eq. (2.5), Eq. (2.10) gives the frequency distribution of equal spacing. The difference between two successive eigenfrequencies is calculated from (2.11) and is shown at the top of Fig. 6 by the solid line. The unit of frequency is given by $\beta / 2 \pi a$, where $\beta$ is the $S$-wave velocity and $\alpha=\sqrt{3} \beta$ is assumed. As expected from (2.10), the difference is nearly constant and its value agrees with the inverse of the travel time of $P$-wave for the radial distance of the sphere.

\section{Spheroidal Oscillation of a Homogeneous Elastic Sphere: Ray Theory}

In this section we apply the ray theory to deriving frequency equations of the spheroidal oscillation of a homogeneous elastic sphere. In the following section, we shall compare these equations with those obtained by the normal mode theory.

P- and S-rays in the sphere which have the phase velocity $C^{\prime}$ (apparent velocity) along the surface obey the relation

$$
C^{\prime}=\alpha / \sin \theta_{p}=\beta / \sin \theta_{s},
$$

where, $\alpha$ and $\beta$ are the $P$ - and $S$-wave velocities, $\theta_{p}$ and $\theta_{s}$, the incident angles of $\mathrm{P}_{\text {- }}$ and $\mathrm{S}$-waves at the surface of the sphere respectively. On the other hand, it is well known that a normal mode solution can be expressed by the superposition of two traveling waves in the opposite colatitudinal directions 
having the phase velocity approximated as

where

$$
C=a \omega / \nu,
$$

$$
\nu=n+1 / 2 \text {. }
$$

$n$ is the colatitudinal order number and $a$ the radius of the sphere. Some comments on the formula (3.2) and the meaning of $n$ are given by Matumoto and SATÔ (1954).

By analogy with the relation between modes and rays appearing in plane boundary problems, it is natural to assume that

Putting

$$
C=C^{\prime} \text {. }
$$

$$
\xi=a \omega / \alpha, \quad \eta=a \omega / \beta,
$$

and combining Eqs. (3.1), (3.2) and (3.4), we have

$$
\sin \theta_{p}=\nu / \xi, \quad \sin \theta_{s}=\nu / \eta \text {. }
$$

In the ray theory, there are three different situations of wave propagation in the sphere, which depend on the value of $C$. In the following we must treat each case separately.

\section{$3.1 C<\beta$}

It is found from (3.1) that $\theta_{p}$ and $\theta_{s}$ have to be complex numbers and thus, in the ordinary sense, there are no body waves corresponding to the normal modes having the phase velocity in this range. The numerical results computed by USAMI and SATÔ (1964) show that the fundamental mode satisfies this condition at high frequencies. SATÔ and USAMI (1962) have proved that at high frequency limits the frequency equation of the spheroidal oscillation can be reduced to a form identical to that of the characteristic equation which gives the velocity of the Rayleigh wave in a semi-infinite medium. IsHII and ELLIS (1970) have shown that the Rayleigh wave can be expressed by using complex angles for propagation directions of plane waves.

\section{$3.2 \beta<C<\alpha$}

This condition is equivalent to $\sin ^{-1}(\beta / \alpha)<\theta_{8}<\pi / 2$, and thus $\mathrm{S}$-waves are always totally reflected and ordinary $\mathbf{P}$-waves do not exist. Here, we develop the discussion as in section 2. Suppose that the sphere is subject to a steady state vibration, two points $\mathrm{A}$ and $\mathrm{B}$ in Fig. 4 are equivalent to each other, except for the phase difference of $a \omega \Delta_{\mathrm{a}} / C$, where $\Delta_{\mathrm{g}}$ is the angular distance between $\mathrm{A}$ and $\mathrm{B}$. Then, the oscillations at $\mathrm{A}$ and $\mathrm{B}$ can be described as $\exp \left\{i \omega\left(t+a \Delta_{s} / C\right)\right\}$ and $\exp (i \omega t)$ respectively, the amplitudes at two points being equally normalized to be unity. According to the ray theory, on the 


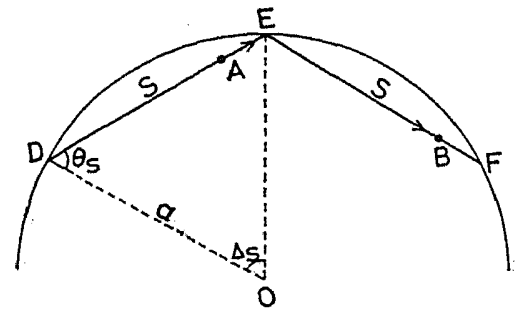

Fig. 4. Ray geometry for a multiply reflected S-wave. A and B are geometrically equivalent points.

other hand, the disturbance at $\mathrm{A}$ propagates with the velocity $\beta$ along the ray path AEB. Then the disturbance at $\mathbf{B}$ is expressed as $\exp \left[i\left\{\omega\left(t+a \Delta_{s} / C-\right.\right.\right.$ $\left.\left.\left.2 a \cos \theta_{s} / \beta\right)+\delta+\pi / 2\right\}\right]$. The term $2 a \cos \theta_{s} / \beta$ is a time delay due to traveling of disturbance as $\mathrm{S}$-wave from $\mathrm{A}$ to $\mathrm{B}$, and $\delta$ and $\pi / 2$ are phase changes due to the reflection at the surface. An interference condition is given by putting this disturbance to be equal to $\exp (i \omega t)$, and we get

$$
1=\exp \left[i\left\{\omega\left(a \Delta_{s} / C-2 a \cos \theta_{s} / \beta\right)+\delta+\pi / 2\right\}\right] .
$$

$\delta$ is a phase shift resulting from total reflection of S-wave at $E$, and $\pi / 2$ is a phase shift inherent in reflected waves in the sphere. SHImamura and SATo (1965) state that each component of the spectrum of a reflected wave changes the phase by $\pi / 2$ for each crossing of two adjacent rays close at the source. The divergence of the wave front in the azimuthal direction produces an effect on both amplitude and phase. This effect is, however, the same for both body waves and waves propagating along the surface, and it does not appear in (3.7).

Provided that the wavelength of waves under consideration is sufficiently short compared with the radius of the sphere, we can regard the wave front and the surface of the sphere as a plane. Then, according to EwING et al. (1957), $\delta$ can be given by the following formula.

$$
\tan (\delta / 2)=-\left\{2-(C / \beta)^{2}\right\}^{2} /\left[4 \sqrt{1-(C / \alpha)^{2}} \sqrt{(C / \beta)^{2}-1}\right] .
$$

Employing Eqs. (3.1) through (3.8) and the relation $\Delta_{s}=\pi-2 \theta_{s}$, we have

$$
\tan \left\{\sqrt{\eta^{2}-\nu^{2}}+\nu \sin ^{-1}(\nu / \eta)-\nu(\pi / 2)-\pi / 4\right\}=-\left(\eta^{2}-2 \nu^{2}\right)^{2} /\left(4 \nu^{2} \sqrt{\eta^{2}-\nu^{2}} \sqrt{\nu^{2}-\xi^{2}}\right) .
$$

When $\eta \gg \nu,(3.9)$ is reduced to

$$
\cos \{\eta-(n+1) \pi / 2\}=0,
$$

which yields equally spaced eigenfrequencies for any assigned value of $n$.

\section{$3.3 C>\alpha$}

In this case both P- and S-waves appear in the sphere, and they are reflected and converted to each other unlimitedly. As is in the previous case, 


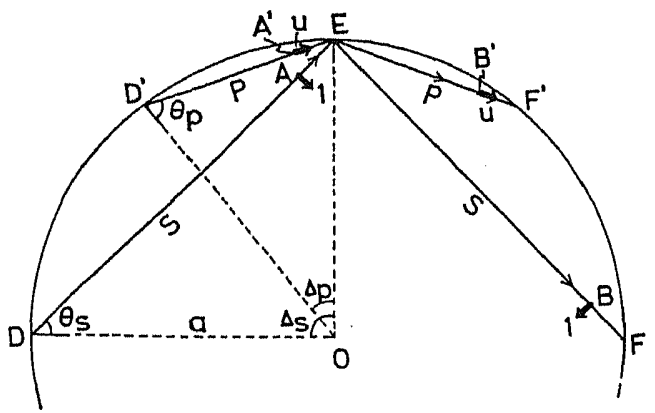

Fig. 5. Ray geometry for multiply reflected and converted P- and S-waves. $A$ and $B, A^{\prime}$ and $B^{\prime}$ are geometrically equivalent points. Amplitudes of displacement of $\mathrm{P}$ - and $\mathrm{S}$-waves are taken as $u$ and 1 respectively.

points $A$ and $B$, and points $A^{\prime}$ and $B^{\prime}$ are equivalent respectively under a steady state vibration (see Fig. 5). This is the same as saying that the amplitudes of a displacement at $\mathbf{A}$ and $\mathbf{B}$ are the same, which we normalize as unity (the direction of the displacement component is defined by the arrow in Fig. 5). Similarly, the amplitudes of $\mathbf{P}$-waves are both taken as $u$ at $\mathbf{A}^{\prime}$ and $\mathbf{B}^{\prime}$. In order that this situation be realizable, the amplitude ratio of $\mathrm{P}$ - and $\mathrm{S}$ waves, $u$, must have a special value depending on the angle of incidence and the wavelength as mentioned by TolstoY and USDIN (1953) for a plane stratified medium. If $A$ and $A^{\prime}$ (similarly $B$ and $B^{\prime}$ ) are taken sufficiently close to the surface of the sphere, we have the following equations for the disturbances at $B$ and $B^{\prime}$ respectively.

$$
\begin{aligned}
& 1=R_{g 8} \mathrm{e}^{i\left(\omega\left(a \Delta_{g} / C-2 a \cos \theta_{8} / \beta\right)+\tilde{\delta}_{88}\right\}}+u R_{p 8} \mathrm{e}^{\left.i(\omega)\left(a \Delta_{g} / C-2 a \cos \theta_{g} / \beta\right)+\delta_{p s}\right\}} \\
& u=u R_{p p} \mathrm{e}^{i\left\{\omega\left(a \Delta_{p} / C-2 a \cos \theta_{p} / \alpha\right)+\delta p p\right\}}+R_{s p} \mathrm{e}^{i\left\{\omega\left(a \Delta_{p} / C-2 a \cos \theta_{p} / \alpha\right)+\delta_{g p}\right\}}
\end{aligned}
$$

where

$$
\Delta_{p}=\pi-2 \theta_{p}, \quad \Delta_{s}=\pi-2 \theta_{s} .
$$

The first and second equations of (3.11) correspond to the disturbances arriving at $\mathrm{B}$ as $\mathrm{S}$-wave and at $\mathrm{B}^{\prime}$ as $\mathrm{P}$-wave respectively. $R_{s 8}, R_{p s}, R_{p p}$ and $R_{8 p}$ are reflection coefficients, and $\delta_{s 8}, \delta_{p s}, \delta_{p p}$ and $\delta_{s p}$ are the phase changes arizing from reflection and conversion of waves in the sphere. Reflection coefficients are defined as the amplitude of the displacement of reflected waves when a wave of unit amplitude is incident on the surface (components are shown by arrows in Fig. 5). Provided that the plane wave approximation is valid, those are given by

$$
\begin{aligned}
R_{s s} & =\left\{\sin 2 \theta_{p} \sin 2 \theta_{s}-(\alpha / \beta)^{2} \cos ^{2} 2 \theta_{s}\right\} / \Delta, \\
R_{p s} & =-(2 \alpha / \beta) \sin 2 \theta_{p} \cos 2 \theta_{s} / \Delta, \\
R_{s p} & =(2 \alpha / \beta) \sin 2 \theta_{s} \cos 2 \theta_{s} / \Delta, \\
R_{p p} & =R_{s s}, \quad R_{s s}^{2}=1+R_{p s} R_{s p}, \\
\Delta & =\sin 2 \theta_{p} \sin 2 \theta_{s}+(\alpha / \beta)^{2} \cos ^{2} 2 \theta_{s} .
\end{aligned}
$$

These relations are at some points different from those used by ToLsToY and 
UsDin (1953). The difference comes from the fact that they have defined the coefficients for vertical components of the displacement. Referring to the paper of ShImamuRa and Sato (1965), we define the phase terms as

$$
\delta_{s s}=\delta_{p s}=\delta_{p p}=\delta_{s p}=\pi / 2 \text {. }
$$

In deriving Eq. (3.11), we have neglected the effect of the phase change due to crossing of rays adjacent in the azimuth, which was pointed out by JeFFREYS and LAPWOOD (1957), because under such a situation, a disturbance traveling along the surface of the sphere undergoes the phase shift of the same quantity $(\pi / 2)$ like the so called polar phase shift.

Eliminating $u$ from the two equations of (3.11), and using Eqs. (3.1) through (3.6), (3.13) and (3.14), we arrive at

where

$$
1-R_{s s} \mathrm{e}^{-2 i X}-R_{s s} \mathrm{e}^{-2 i Y}+\mathrm{e}^{-2 i(X+Y)}=0,
$$

$$
\begin{aligned}
X & =\sqrt{\xi^{2}-\nu^{2}}+\nu \sin ^{-1}(\nu / \xi)-\nu \pi / 2-\pi / 4 \\
Y & =\sqrt{\eta^{2}-\nu^{2}}+\nu \sin ^{-1}(\nu / \eta)-\nu \pi / 2-\pi / 4 \\
R_{s s} & =\left\{4 \nu^{2} \sqrt{\eta^{2}-\nu^{2}} \sqrt{\xi^{2}-\nu^{2}}-\left(\eta^{2}-2 \nu^{2}\right)^{2}\right\} /\left\{4 \nu^{2} \sqrt{\eta^{2}-\nu^{3}} \sqrt{\xi^{2}-\nu^{2}}+\left(\eta^{2}-2 \nu^{2}\right)^{2}\right\} .
\end{aligned}
$$

Equation (3.15) can be simplified as

or as

$$
\cos (X+Y)-R_{s s} \cos (X-Y)=0
$$

$$
4 \sqrt{(\eta / \nu)^{2}-1} \sqrt{(\xi / \nu)^{2}-1} \tan X \tan Y-\left\{(\eta / \nu)^{2}-2\right\}^{2}=0 .
$$

Equation (3.17) has a form similar to (2.5) but is much complicated, and it is difficult to discuss general properties of its solutions.

In the case when $\eta$ is infinitely large, (3.17) can be reduced to

$$
\cos \{\xi-(n+1) \pi / 2\} \cos \{\eta-(n+1) \pi / 2\}=0 \text {. }
$$

According as $n$ is even or odd, this is rewritten as

$$
\sin \xi \sin \eta=0 \text { ( } n \text { : even), } \quad \cos \xi \cos \eta=0 \text { ( } n \text { : odd }) .
$$

Equation (3.19) or (3.20) means that frequency equations for P-wave and S-wave are decoupled under the condition that $\eta \gg \nu$, which is satisfied by the rays with small incident angles (see Eq. (3.6)). This decoupling follows from the fact that little conversion from $\mathrm{P}$ to $\mathrm{S}$ or $\mathrm{S}$ to $\mathrm{P}$ occurs for waves associated with such rays (see Eq. (3.13)) and thus the P- and S-waves behave independently in the sphere. Gilbert (1975) shows that the decoupling of P-and Swaves is generally possible in a radially heterogeneous spherical earth.

Equation (3.20) can be derived by the method developed in section 2 . In this case, the dependence of the equation on $n$ comes from the fact that displacements in the sphere associated with the order number $n$ are spherically symmetric or anti-symmetric according as $n$ is even or odd. Hence, when $n$ 
is an even number, we have the equation similar to $(2.10)$ for both $P_{\text {- }}$ and $\mathrm{S}$-waves. When $n$ is an odd number, the negative sign have to be taken in the right side of (2.8). This method is superior to the previous one in that there is no assumption as to the value of $n$. As a consequence, Eq. (3.20) holds for all positive values of $n$.

In Fig. 6, the solid curves denote the difference between two successive frequencies, ${ }_{i+1} \eta_{n}-{ }_{i} \eta_{n}$, as plotted against $i$, where ${ }_{i} \eta_{n}$ is the eigenfrequency of the $i$-th radial and $n$-th colatitudinal mode and is calculated from an exact frequency equation. $i$ is numbered in the usual way for a given $n$, that is, in the order of increasing frequency. In the high frequency range where the decoupling of $\mathrm{P}$ and $\mathrm{S}$ is possible, eigenfrequencies thus defined comprise two kinds of independent solutions, one being concerned with $\mathrm{P}$-waves and the other with S-waves. The oscillatory nature of the solid curves is apparently caused by such a mixed composition of frequencies, and thus is not called the solotone effect, though the curves are similar to the ones in Fig. 3. Once the frequencies are decoupled, those of each group are distributed with equal
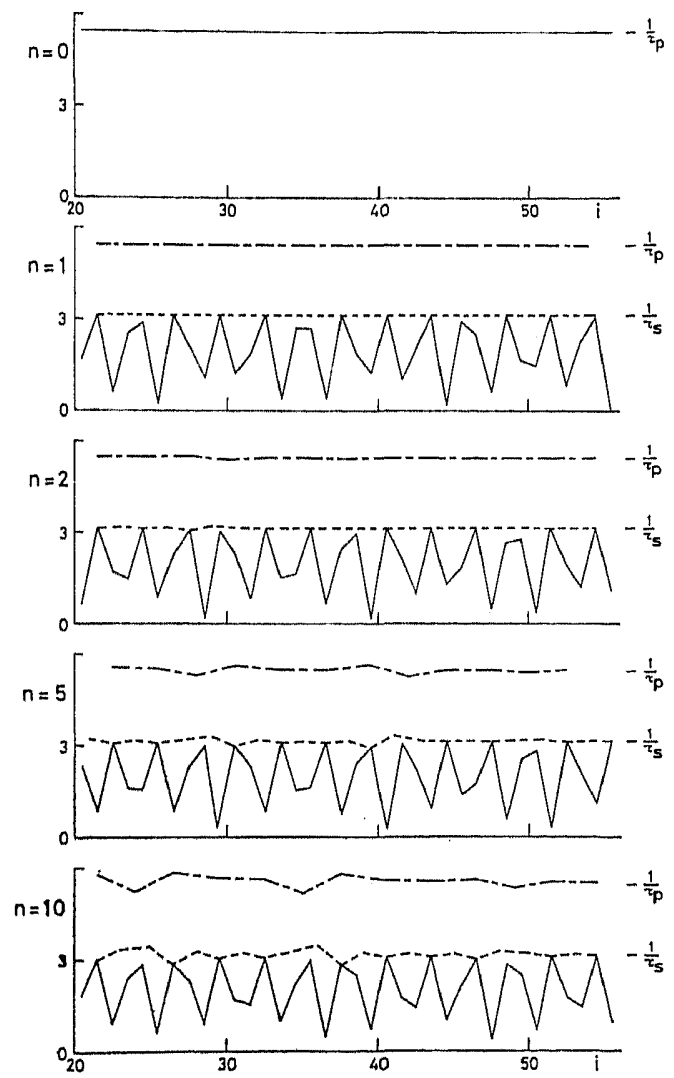

Fig. 6. Difference of two successive spheroidal eigenfrequencies for a homogeneous sphere as plotted against the radial mode number $i$. Chain and dashed curves correspond to the frequencies decoupled as P- and S-group respectively and solid curves to all the frequencies. 


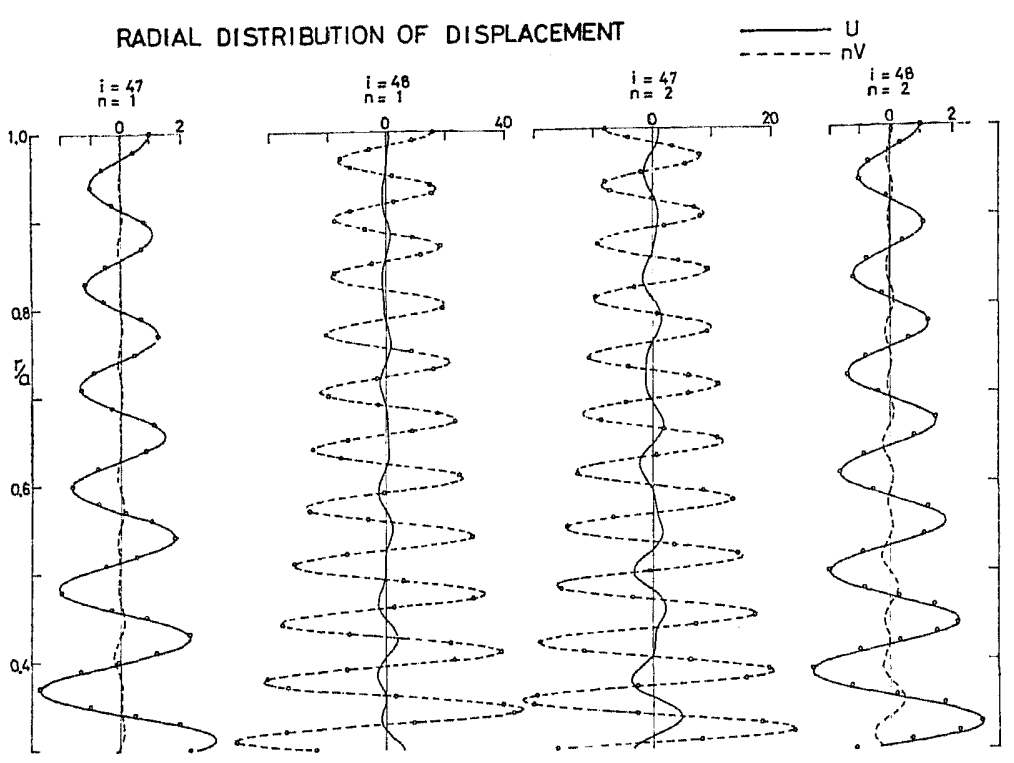

Fig. 7. Radial and colatitudinal displacement, $U$ and $n V$, for the spheroidal modes of a homogeneous sphere as a function of radius.

spacing. The chain and dashed curves are the results calculated for two groups of the decoupled frequencies, P- and S-group, respectively. $\tau_{p}$ and $\tau_{s}$ are the travel times of $\mathrm{P}$ - and $\mathrm{S}$-waves required for crossing the sphere through its center. When $n$ is small, decoupling is perfect even for low radial modes. For large $n$, however, computations up to larger mode numbers are necessary for the perfect decoupling of the two groups.

Well-decoupled two modes also possess two distinctive radial dependences of displacement. Figure 7 shows such radial distributions of radial (solid) and colatitudinal (dashed) displacements associated with two successive radial modes. The radial displacement at the surface of the sphere is normalized to be unity. The outside figures show two modes belonging to the P-group and the inside two figures show two modes belonging to the Sgroup. The radial component is predominant for the $\mathrm{P}$-group modes, having a property inherent in $\mathrm{P}$-waves traveling in the radial direction. On the contrary, the colatitudinal component is prominent in the S-group modes. This property is consistent with that of S-waves traveling in the radial direction. As inferred from the figure, the radial mode number $i$ is not an appropriate indicator of the number of nodal surfaces of radial functions, and a new notation should be adopted for each group of the modes separately, as mentioned by ANDERSSEN et al. (1975). Figures 6 and 7 are reproduced from a paper by ODAKA (1977). 


\section{Spheroidal Oscillation of a Homogeneous Sphere: Normal Mode Theory}

The frequency equation for the present model can be written in a compact form (ODAKA and USAMI, 1978) as

$$
g\left(j_{n}, h a\right)\left\{f\left(j_{n}, k a\right)+g\left(j_{n}, k a\right)\right\}-n(n+1) f\left(j_{n}, h a\right) f\left(j_{n}, k a\right)=0,
$$

where

$$
\begin{aligned}
& f\left(j_{n}, \zeta r\right)=(2 / r) \dot{j_{n}}(\zeta r)-\left(2 / r^{2}\right) j_{n}(\zeta r), \\
& g\left(\dot{j}_{n}, \zeta r\right)=-(4 / r) \dot{j_{n}}(\zeta r)+\left\{2 n(n+1) / r^{2}-k^{2}\right\} j_{n}(\zeta r), \quad(\zeta=h \text { or } k) .
\end{aligned}
$$

The dot on the spherical Bessel function $j_{n}(\zeta r)$ stands for $\mathrm{d} / \mathrm{d} r . h$ and $k$ are the wave numbers for $\mathrm{P}$ - and $\mathrm{S}$-waves respectively and are connected with $\xi$ and $\eta$ of (3.5) as follows.

$$
h a=\xi, \quad k a=\eta .
$$

In this section, we investigate asymptotic expressions of Eq. (4.1), which possess different forms corresponding to respective cases of section 3 .

\section{$4.1 \nu>\eta$}

Referring to Eqs. (3.2) and (3.5), it is found that the above condition is equivalent to $C<\beta$. SATÔ and USAMI (1962) have already discussed the present case with the use of the following asymptotic expressions for Bessel functions valid for large values of $\nu$.

$$
\begin{aligned}
J_{\nu}(\nu \operatorname{sech} x)= & \exp \{\nu(\tanh x-x)\} / \sqrt{2 \pi \nu \tanh x}, \\
J_{\nu+1}(\nu \operatorname{sech} x)= & \exp \{\nu(\tanh x-x)-x\} /[2 \pi\{(\nu+1) \tanh x \\
& \left.\left.+\operatorname{sech}^{2} x \operatorname{coth} x\right\}\right]^{1 / 2}, \quad(x>0) .
\end{aligned}
$$

They finally reduced (4.1) to

$$
\left[2-(\eta / \nu)^{2}\right]^{2}-4 \sqrt{1-(\eta / \nu)^{2}} \sqrt{1-(\eta / \nu)^{2}(h / k)^{2}}=0 .
$$

This equation has an identical form with that of the characteristic equation for Rayleigh waves in a semi-infinite medium.

\section{$4.2 \xi<\nu<\eta$}

This condition is equivalent to $\beta<C<\alpha$. The inequality between $\nu$ and $\xi$ is the same for cases 4.1 and 4.2 , and thus (4.4) is still available for reducing the terms such as $j_{n}(\xi)$ and $j_{n+1}(\xi)$ in (4.1). For a function of $\eta$, however, the following asymptotic formulas have to be introduced,

$$
\begin{gathered}
J_{\nu}(\nu \sec y)=\cos \{\nu(\tan y-y)-\pi / 4\} / \sqrt{(\pi / 2) \nu \tan y}, \\
J_{\nu-1}(\nu \sec y)=\cos \{\nu(\tan y-y)-\pi / 4+y\} /\{(\pi / 2)(\nu \tan y+\cot y)\}^{1 / 2}, \\
(0<y<\pi / 2) .
\end{gathered}
$$


which have been used by ODAKA (1972) in obtaining radiation patterns of toroidal modes for a double couple source. Putting

$$
\xi=\nu \operatorname{sech} x, \quad \eta=\nu \sec y,
$$

and employing (4.4) and (4.6), we have

$$
\begin{aligned}
& j_{n+1}(\xi) / j_{n}(\xi)=\exp (-x) / \sqrt{1+(1 / \nu) \operatorname{coth}^{2} x}, \\
& j_{n-1}(\eta) / j_{n}(\eta)=[\cos y-\sin y \cdot \tan \{\nu(\tan y-y)-\pi / 4\}] / \sqrt{1+(1 / \nu) \cot ^{2} y} .
\end{aligned}
$$

With the aid of (4.8) and the recurrence formulas for the spherical Bessel functions, Eq. (4.1) can be reduced to

where

$$
\begin{gathered}
4 \nu^{2} \sqrt{\eta^{2}-\nu^{2}} \sqrt{\nu^{2}-\xi^{2}} \tan Y+\left(\eta^{2}-2 \nu^{2}\right)^{2}+2\left(2 \eta^{2}-\nu^{2}\right) \sqrt{\nu^{2}-\xi^{2}} \\
-2\left(\eta^{2}+\nu^{2}\right) \sqrt{\eta^{2}-\nu^{2}} \tan Y=0,
\end{gathered}
$$

$$
Y=\nu(\tan y-y)-\pi / 4 \text {. }
$$

Eliminating from (4.9) the terms of lower order in $\eta$ and $\nu$, we get

$$
4 \nu^{2} \sqrt{\eta^{2}-\nu^{2}} \sqrt{\nu^{2}-\xi^{2}} \tan \left\{\sqrt{\eta^{2}-\nu^{2}}-\nu y-\pi / 4\right\}+\left(\eta^{2}-2 \nu^{2}\right)^{2}=0 \text {. }
$$

Referring to (3.6) and (4.7), it is readily found that Eq. (4.10) is identical to (3.9). When $\eta \gg \nu$, we have to return to (4.1). Then, keeping the predominant terms (terms of the highest order in $\eta$ ), we can get the same equation as (3.10).

\section{$4.3 \nu<\xi$}

This condition is equivalent to $C>\alpha$. In this case, the first relation of (4.7) must be replaced by

$$
\xi=\nu \sec x
$$

and asymptotic formulas like (4.6) have to be used for both functions of $\xi$ and $\eta$. After some manipulations, we get

where

$$
\begin{gathered}
4 \nu^{2} \sqrt{\xi^{2}-\nu^{2}} \sqrt{\eta^{2}-\nu^{2}} \tan X \tan Y-\left(\eta^{2}-2 \nu^{2}\right)^{2}+2\left(2 \eta^{2}-\nu^{2}\right) \sqrt{\xi^{2}-\nu^{2}} \tan X \\
+2\left(\eta^{2}+\nu^{2}\right) \sqrt{\eta^{2}-\nu^{2}} \tan Y=0,
\end{gathered}
$$

$$
X=\nu(\tan x-x)-\pi / 4, \quad Y=\nu(\tan y-y)-\pi / 4 .
$$

Provided that the third and fourth terms in (4.12) are negligibly small compared with other terms, the equation can be simplified as

$$
\begin{gathered}
4 \nu^{2} \sqrt{\xi^{2}-\nu^{2}} \sqrt{\eta^{2}-\nu^{2}} \tan \{\nu(\tan x-x)-\pi / 4\} \tan \{\nu(\tan y-y)-\pi / 4\} \\
-\left(\eta^{2}-2 \nu^{2}\right)^{2}=0 .
\end{gathered}
$$

It is easily proved that (4.13) is identical to (3.18).

The above condition is generally satisfied when the orders of magnitude of $\eta, \xi$ and $\nu$ are equal to each other. When $\eta$ is very large compared with $\nu$, 
we have to return to (4.1). Then, keeping terms of the highest order in $\eta$, we get

$$
\cos \{\nu(\tan x-x)-\pi / 4\} \cos \{\nu(\tan y-y)-\pi / 4\}=0,
$$

which is equivalent to (3.19).

Alternatively, employing the asymptotic formula of the spherical Bessel function valid for a large argument and applying it to the function $j_{n}(\xi) j_{n}(\eta)$, we immediately have Eq. (3.19).

\section{Discussion and Conclusion}

The ray theory has proved successful in obtaining frequency equations of the radial oscillation of a homogeneous sphere and a one-layered sphere (a sphere with one discontinuous surface in it) and the spheroidal oscillation of a homogeneous sphere. Formulation is made on the basis of a certain interference condition of body waves traveling in the sphere. It is proved that equations thus obtained agree with the asymptotic frequency equations derived by the normal mode theory. It should be noted that the equations based on the ray theory and normal mode theory are consistent only at high frequencies in spherical problems, whereas the two approaches lead to identical equations in flat boundary problems. If we use reflection and transmission coefficients valid for a spherical boundary, an improved relation may be found between two results.

The method used for deriving the asymptotic frequency equation of the radial oscillation of the one-layered sphere in terms of ray theory makes it clear that the solotone effect is connected with multiple reflections of body waves at the discontinuity in the medium.

Asymptotic properties of spheroidal overtones of the homogeneous sphere are studied and decoupling of $\mathrm{P}_{\text {- }}$ and $\mathrm{S}$-waves at high frequencies is well illustrated in relation to the distribution of eigenfrequencies and the radial dependence of eigenfunctions. Further discussion on the connection betwreen modes and rays is made by ODAKA and USAMI (1978), who calculate surface displacements by the ray theory in order to compare them with mode solutions, and confirm the validity of Eq. (3.11).

The ray theoretical method developed in this paper can be applied to other cases such as the torsional oscillation of a spherical shell, which is analytically treated by SATO and LAPWOOD (1977). However, when a number of discontinuities in the sphere increases, more rays must be taken into consideration and thus this method becomes much more complicated and practically impossible.

The mode-ray correspondence relation has become clearer through the present investigation. It must, however, be noted that many normal modes 
besides those satisfying relation (3.4) must be taken into account in order to construct actual body wave phases in the synthesis of theoretical seismograms, as shown by ODAKA and USAMr (1972).

After completing this work, the author found that BRUNE (1966) had already derived the asymptotic frequency equation of the spheroidal oscillation of a homogeneous sphere in a similar way. Brune's attention is devoted to investigating the relation between travel times of body waves and dispersion curves of normal modes, while this paper is concerned with discussing the frequency equation in detail in terms of the ray theory and normal mode theory and investigating its asymptotic properties.

The author wishes to thank Professor Tatsuo Usami, Earthquake Research Institute, University of Tokyo, for kindly reading the manuscript and making useful suggestions. The author is also grateful to Dr. E. R. Lapwood, who made many helpful suggestions and made his own manuscript available before publication.

\section{REFERENCES}

Anderssen, R. S., J. R. Cleary, and A. M. Dziewonski, Asymptotic structure in eigenfrequencies of spheroidal normal modes of the Earth, Geophys. J. R. Astron. Soc., 43, 1001$1005,1975$.

Ben-Menahem, A., Mode-ray duality, Bull. Seismol. Soc. Am., 54, 1315-1321, 1964.

Brune, J. N., Travel times, body waves, and normal modes of the Earth, Bull. Seismol. Soc. Am., 54, 2099-2128, 1964.

BRUNe, J. N., P and $\mathrm{S}$ wave travel times and spheroidal normal modes of a homogeneous sphere, J. Geophys. Res., 71, 2959-2965, 1966.

EwIng, W. M., W. S. JARDetzKY, and F. Press, Elastic Waves in Layered Media, McGrawHill, 1957.

GiLbert, F., Some asymptotic properties of the normal modes of the Earth, Geophys. J. R. Astron. Soc., 43, 1007-1011, 1975.

IsHII, H. and R. M. Ellis, Expression of a free Rayleigh wave using complex angles, Sci. Rep. Tohoku Univ. Ser. 5, Geophys., 20, 45-47, 1970.

Jeffreys, H. and E. R. Lapwood, The reflection of a pulse within a sphere, Proc. $R$, Soc. London A, 241, 455-479, 1957.

LAPWOOD, E. R., The effect of discontinuities in density and rigidity on torsional eigenfrequencies of the Earth, Geophys. J. R. Astron. Soc., 40, 453-464, 1975.

Matumoto, T. and Y. SATô, On the vibration of an elastic globe with one layer. The Vibration of the first class, Bull. Earthq. Res. Inst., 32, 247-258, 1954.

MCNABB, A., R. S. ANDERSSEN, and E. R. LAPwood, Asymptotic behavior of the eigenvalues of a Sturm-Liouville system with discontinuous coefficients, J. Math. Anal. Appl., 54, 741-751, 1976.

ODAKA, T., On azimuthal variation of displacements of normal mode solutions of SH type excited by a double-couple point source with special attention to the mode-ray relation, J. Phys. Earth, 20, 71-87, 1972.

ODAKA, T., Some properties of higher mode oscillations of a homogeneous elastic sphere, Zisin 2, 30, 118-122, 1977 (in Japanese).

ODAKA, T., Radial oscillations of a multi-layered elastic sphere and theoretical seismograms: 
Effects of surface layers on waveform of P waves, Bull. Earthq. Res. Inst., 53, 249-264, 1978 (in Japanese).

ODAKA, T. and T. USAMI, On the correspondence relation between normal modes and rays: Case of torsional oscillation of a spherical earth with a homogeneous mantle and a liquid core, J. Phys. Earth, 20, 89-109, 1972.

ODAKA, T. and T. UsAmI, Some properties of spheroidal modes of a homogeneous elastic sphere with special reference to radial dependence of displacement, submitted to $J$. Comput. Phys. (Zipora Alterman Memorial Number), 1978.

OfFICER, C. B., Normal mode propagation in three layered liquid half-space by ray theory, Geophysics, 16, 207-212, 1951.

Press, F. and M. Ewing, A theory of microseisms with geologic applications, Trans. Am. Geophys. U., 29, 163-174, 1948.

SATO, R. and E. R. LAPwOOD, The asymptotic distribution of torsional eigenfrequencies of a spherical shell. I, J. Phys. Earth, 25, 257-282, 1977.

SAtô, Y., Study on surface waves I. Velocity of Love waves, Bull. Earthq. Res. Inst., 29, 1-11, 1951.

SATÔ, Y. and T. USAMI, Basic study on the oscillation of a homogeneous elastic sphere II. Distribution of displacement, Geophys. Mag., 31, 25-47, 1962.

Shimamura, H. and R. SAto, Model experiments on body waves-travel times, amplitudes, wave forms and attenuation, J. Phys. Earth, 13, 10-33, 1965.

Tolstoy, I., Dispersive properties of a fluid layer overlying a semi-infinite elastic solid, Bull. Seismol. Soc. Am., 44, 493-512, 1954.

Tolstoy, I. and E. Usdin, Dispersive properties of stratified elastic and liquid media: A ray theory, Geophysics, 18, 844-870, 1953.

UsamI, T. and Y. SATô, Propagation of spheroidal disturbances on a homogeneous elastic sphere, Bull. Earthq. Res. Inst., 42, 273-287, 1964.

WANG, C., J. F. GetTrust, and J. R. Cleary, Asymptotic overtone structure in eigenfrequencies of torsional normal modes of the Earth: A model study, Geophys. J.R. Astron. Soc., 50, 289-302, 1977. 